\title{
Hydrodynamic Study of Power-Law Fluids across Unconfined Semi- Circular Cylinder at Low Reynolds Numbers: Effect of Orientation angle
}

\author{
Jaspinder Kaur, Anurag Kumar Tiwari, Jatinder Kumar Ratan \\ Department of Chemical Engineering, National Institute of Technology \\ Jalandhar-144011, India \\ jaspinderkp@gmail.com, tiwaria@nitj.ac.in, kumarj@nitj.ac.in
}

\begin{abstract}
The flow of a non-Newtonian, power-law fluids past a semi-circular cylinder is considered in this present study. The governing differential equations for fluid flow have been solved numerically at low Reynolds numbers $(R e \leq 1)$. The results are analyzed at different orientation angle of semi-circular cylinder, in terms of velocity profile near the cylinder, shear-rate and total drag coefficient. It is found that at low Reynolds number ( $R e \leq 1)$, drag coefficient shown the inverse classical relation with the Reynolds numbers for shear-thinning fluids $(n<1)$ whereas for shear-thickening fluids $(n>1)$ the drag coefficient tends to become coincide for all orientation angle. Finally, the present numerical values of the total drag coefficient for all orientations in shear-thinning fluids have been correlated via a simple expression.
\end{abstract}

Keywords: Non-Newtonian, power-law fluids, Reynolds numbers, semi-circular cylinder.

\section{Introduction}

The fluid flow from a cylinder of different shape is popular not only because industrial problems associated with process design calculations. In such situations involving fluid flow past a cylinder of various shapes, reliable information about hydrodynamic force are frequently needed for the purpose of process design [1]. Additional applications are found in polymer processing operations where most of the fluids encountered exhibit non-Newtonian fluid behavior. It can be seen that there is no investigation available on hydrodynamic phenomena from a semi-circular cylinder in non- Newtonian power law fluids. In particular, the field equations, namely, mass and momentum governing the present flow phenomena from an semicylinder, have been studied extensively with different orientation angles at low value of Reynolds number $(\operatorname{Re} \leq 1)$.

\subsection{Problem statement and mathematical formulation}

Consider the two-dimensional, unconfined, incompressible and a laminar flow of a power-law fluid flow over a semicircular cylinder at different angle of diameter, $d$. At low Reynolds number, the flow is expected to be steady (time independed). The flow domain, it is not possible to study numerically the unconfined flow condition, it needs to be approximated here by an artificial confine circular domain of diameter $D_{\infty}$ for semi-circular cylinder, as shown in Fig 1 . The value of diameter $D_{\infty}$ of the confining fluid envelope is chosen to be sufficiently large in order to minimize the boundary effects on the flow phenomena while maintaining the required computational effort at a modest level. Since the semicircular cylinder is infinitely long in the z-direction, the velocity component, $V_{z}=0$, and there are no gradients in the in z-direction, i.e., $\partial() / \partial z=0$. 


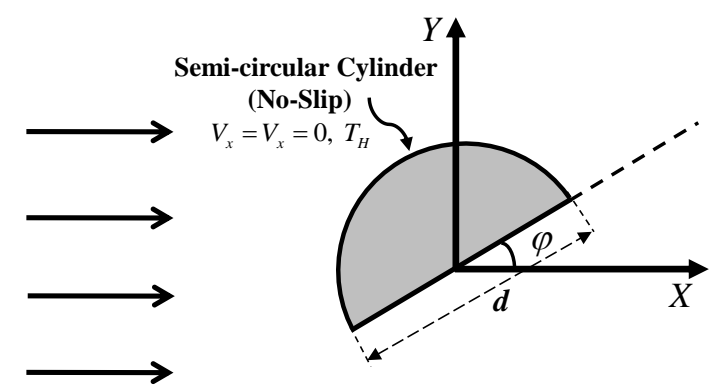

Fig. 1: Schematics of diagram for a semi-circular cylinder.

Within the framework of these assumptions, the governing equations in their dimensionless form can be written as follows:

\section{Continuity equation:}

$$
\frac{\partial V_{x}}{\partial x}+\frac{\partial V_{y}}{\partial y}=0
$$

\section{X-Momentum equation:}

$$
\frac{\partial\left(V_{x} V_{x}\right)}{\partial x}+\frac{\partial\left(V_{x} V_{y}\right)}{\partial y}=-\frac{\partial p}{\partial x}+\frac{1}{\operatorname{Re}}\left(\frac{\partial \tau_{x x}}{\partial x}+\frac{\partial \tau_{y x}}{\partial y}\right)
$$

Y-Momentum equation:

$$
\frac{\partial\left(V_{y} V_{x}\right)}{\partial x}+\frac{\partial\left(V_{y} V_{y}\right)}{\partial y}=-\frac{\partial p}{\partial y}+\frac{1}{R e}\left(\frac{\partial \tau_{x y}}{\partial x}+\frac{\partial \tau_{y y}}{\partial y}\right)
$$

For a power-law incompressible fluid [2], the deviatoric stress components, $\tau_{i j}$, are related to the rate of deformation tensor, $\varepsilon_{i j}$, as follows:

$$
\tau_{i j}=2 \eta \varepsilon_{i j}
$$

Where the component of the rate of deformation tensor are given as:

$$
\varepsilon_{i j}=\frac{1}{2}\left(\frac{\partial V_{i}}{\partial j}+\frac{\partial V_{j}}{\partial i}\right)
$$

For the power law fluid, the scaler viscosity $\eta$ is defined as:

$$
\eta=m\left(I_{2} / 2\right)^{(n-1) / 2}
$$


In Eq. (6), $m$ is flow consistency index and $n$ is the power-law index. For Newtonian fluids, the value of power-law index, i.e., $n=1$; for $n<1$ indicates shear-thinning fluid behavior and for $n>1$ indicates shear-thinning fluid behavior. In Eq. (6), $I_{2}$ is the second invariant of the rate of strain tensor which may be expressed as:

$$
I_{2}=\sum \sum \varepsilon_{i j} \cdot \varepsilon_{i j}=\varepsilon_{x x}^{2}+\varepsilon_{x y}^{2}+\varepsilon_{y x}^{2}+\varepsilon_{y y}^{2}
$$

The modelling part of present study is completed by apply the physically realistic boundary conditions. In brief, the conditions of no-slip on the surface of the cylinder (i.e., $V_{x}=V_{y}=0$ ) were used. The left-half of the surrounding envelope of fluid was designated as the inlet velocity boundary condition $\left(i . e ., V_{x}=1, V_{y}=0\right)$ is used. Similarly, the right-half of the cylindrical envelope of fluid is designated as the outflow boundary condition $\left(i . e ., \partial \varphi / \partial x=0\right.$, where $V_{x}$ and $\left.V_{y}\right)$ as the relevant boundary condition in this work.

The field variables appearing in the aforementioned Eq.(1)-(3) and specified boundary conditions are rendered dimensionless using $D, V_{\infty}, \rho V_{\infty}^{2}$ and $m\left(V_{\infty} / D\right)^{n-1}$ scaling variables for length, velocity, pressure and viscosity, respectively. The dimensionless group, namely, Reynolds number (Re) appearing in Eq.(2) and (3) are defined as follows:

- The Reynolds number (Re) for power-law fluids is defined as follows:

$$
\operatorname{Re}=\frac{\rho_{\infty} V_{\infty}^{2-n} D^{n}}{m}
$$

The numerical solution of flow domain maps in terms of the primitive variables, namely, $V_{x}, V_{y}, p$. These, in turn, are used to deduce the local and global characteristics, it is appropriate to introduce some definitions:

- The Surface pressure coefficient $(C \mathrm{p})$ is defined as follows:

$$
C_{P}=\frac{p_{s}-p_{\infty}}{\frac{1}{2} \rho V_{\infty}^{2}}
$$

Where $p_{s}$ the surface is pressure and $p_{\infty}$ is the free stream pressure at the exit boundary

- The total drag coefficient $\left(C_{\mathrm{D}}\right)$, which is the sum of the friction and pressure components, is defined as

$$
C_{D}=\frac{F_{D}}{\frac{1}{2} \rho V_{\infty}^{2} D}
$$

\section{Numerical solution procedure and computational parameters}

Following the numerical methodology and strategy for arriving at optimum computational suffice it to say here, that the results reported herein are based on the following values: $\mathrm{D} / \mathrm{d}=1500$ and a non-uniform computational mesh consisting of very small size element on the surface $\delta / d=0.0098$, whereas the total number of elements was 76,720 for all orientation angle. A simulation was deemed to have converged when the relative residuals for the momentum equations were lower than 
$10^{-5}$. This was also sufficient to ensure that the drag values had stabilized at least up to four significant digits. The numerical solution and as well as the computational mesh was obtained using the finite element based solver COMSOL Multiphysics [3].

\section{Results and discussion}

The present numerical results for semi-circular cylinder at different angle are shown on flow patterns, pressure coefficient and shear rate and total drag coefficient at low Reynold number range $(0.01 \leq R e \leq 1)$. Before going to the final results of present study, it is imperative to ascertain the reliability and precision of the results obtained in this study. This objective is accomplished here by using results from the prior studies available in the literature for the purpose of benchmarking.

As noted earlier, while there are no prior results available for the present configuration geometry investigated in the following range of condition, reliable results are available for validation is a circular cylinder and this constitutes the benchmark problem for the purpose of general validity of the numerical solution methodology used here. Table 1 , show comparisons between the present and literature results for low value of Reynolds number $(\operatorname{Re}=0.1)$. While the present values differ from that of Pantokratoras [4] by $\sim 3 \%$ (Table 1), the corresponding deviations are of the order of $\sim 1 \%$ from that of Oseen [5]. Based on the comparisons in conjunction with our past experience, the present results for a semi-circular cylinder submerged in power-law fluids are believed to be reliable to within 1-4\%.

Table 1: Comparison of the present results with the literature values for circular cylinder.

\begin{tabular}{llll} 
Re & Pantokratoras [4] & Oseen [5] & Present \\
\hline & & & \\
0.1 & 373.67 & 380.37 & 384.75 \\
0.01 & 55.711 & 58.383 & 59.43 \\
\hline
\end{tabular}

To understand the flow field near the cylinder, it is important to visualised streamline profiles for all orientations at Re $=0.1$ shown in Fig 2. At low value of Reynolds numbers, the fluid inertia force is negligible as compare to viscous forces and therefore a fluid movement over the submerge body due to only viscous effects only. In that case, fluid element is following the contour of the submerged body without much loss in its momentum. In this case of non- Newtonian powerlaw fluids, the viscous force scales as $\sim V^{n}$ whereas the inertial force scales as $\sim V^{2}$. Thus, for a shear-thinning fluid, $(n<1)$, the viscous force shows a weaker dependence on the velocity than that in Newtonian fluids. Due to the no-slip condition on the surface of the cylinder, the rate of deformation of the fluid is high in this region and therefore, a shear-thinning fluid exhibits its minimum viscosity here which progressively increases away from the cylinder. This is tantamount to the fluidlike region being surrounded by a high viscosity solid-like boundary. Such confinement acts to stabilize the flow. It is clearly seen that no flow separation observed at low Reynolds number except orientation $\emptyset=90^{\circ}$ in shear-thickening $n=1.8(n>$ 1). This observation is indicated that the delay in flow separation at orientation $\emptyset=90^{\circ}$ that is consistent with literature results [6-8] 
(A)
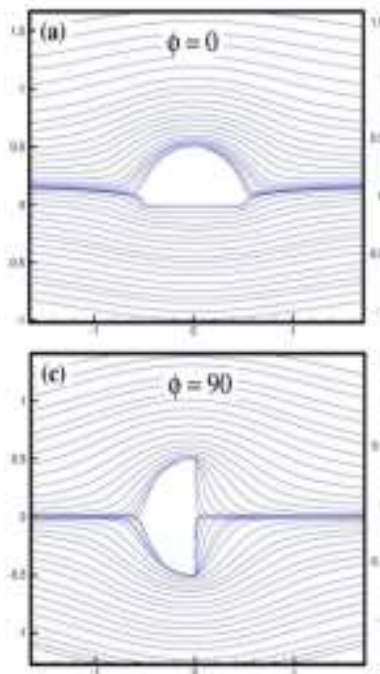
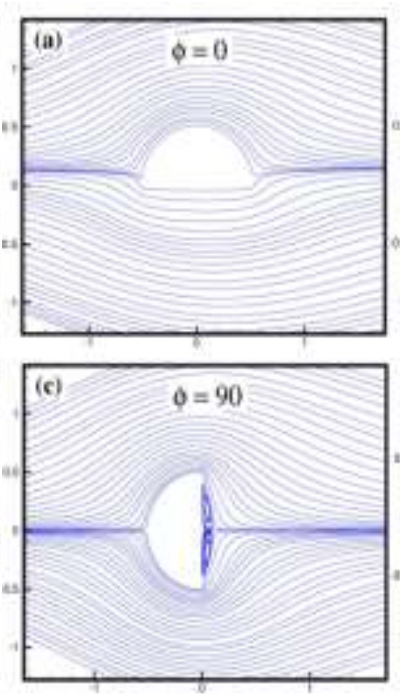

(B)
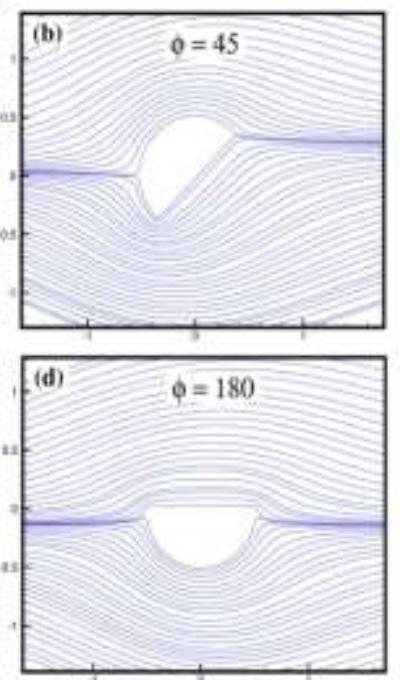

Fig. 2: Streamline profiles (A) for shear-thinning fluids $(\mathrm{n}=0.2)$, (B) shear-thickening fluids $(\mathrm{n}=1.8)$ at $\operatorname{Re}=0.1$.

Additional insights can be gained by examining the variation of dimensionless surface shear rate as shown in Fig 3 for two extreme cases at $\varnothing=0^{\circ}$ and $180^{\circ}$ and for shear-thinning fluids $(n=0.2$, Fig 3(A)) and shear-thickening fluids $(n=1.8$, Fig 3(B))) respectively. It is observed that at low Reynolds number, the variation of shear rates are much higher in shearthinning fluids $(n=0.2)$ than that of shear-thickening, $n=1.8$. The effective viscosity of shear-thinning fluid shown the minimum viscosity near the cylinder which indicates the higher rate of shear. As the Reynolds number change from Re $=$ 0.01 to $\mathrm{Re}=1$ that means the relative strength of inertial increases that translate the higher rate of shear. As for as geometries concerned, maximum and minimum shear rate observed on the mid of the curved surface (ACB) and the flat surface (AB) respectively. The same observations are also true for other orientation angle. 

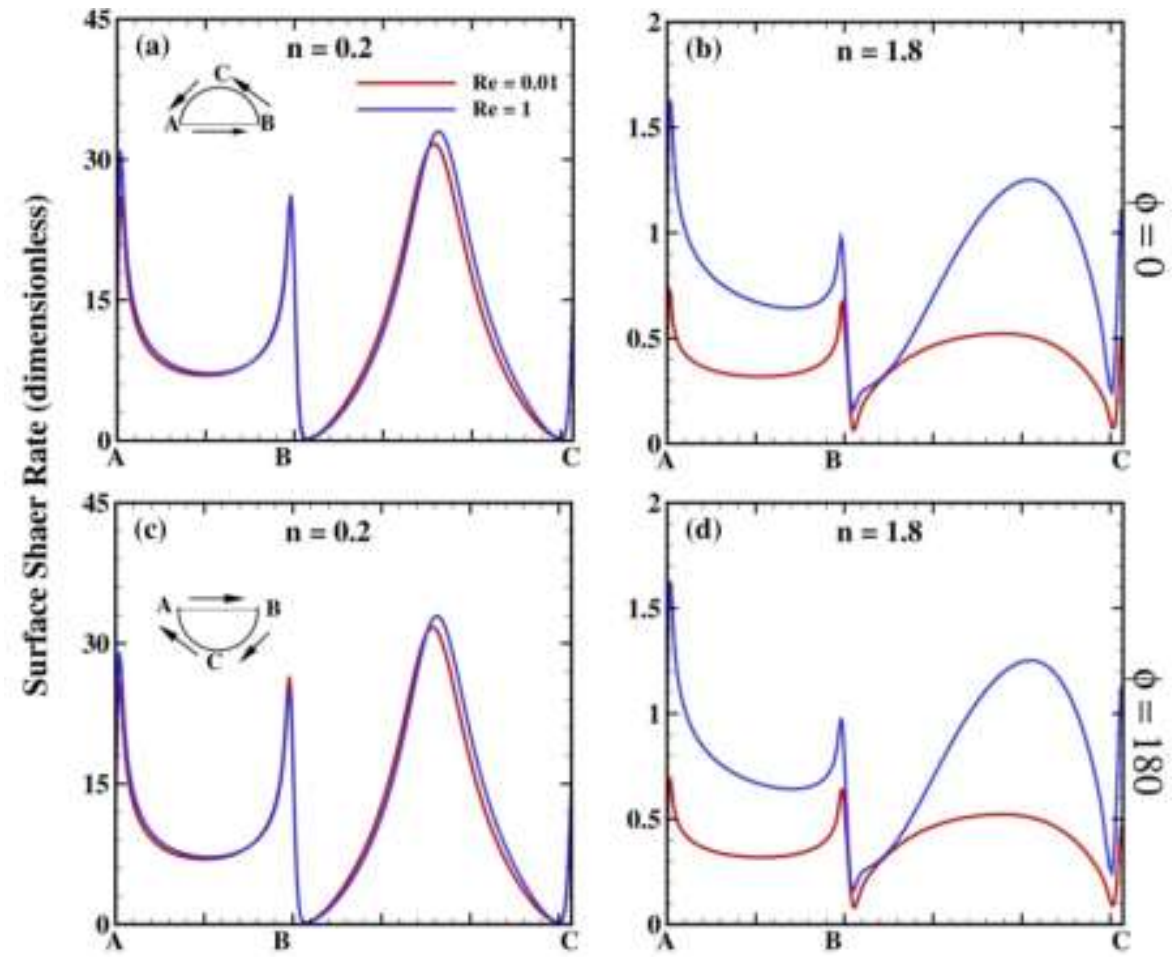

Fig. 3: Variation of surface shear rate $(\dot{\gamma} d / V)$.

Finally, the dependence of the total drag coefficient on orientation of cylinder and power-law index are shown in Fig .4. It is clear that the total drag coefficient is enhanced in shear-thinning fluids $(n<1)$ and it is reduced in shear-thickening $(n>1)$ fluids, at low value of Reynolds numbers irrespective of orientation angle. The magnitude strength of the cylinder at $0^{\circ}$ and $180^{\circ}$ are almost same and that is higher as compare to other orientation angle (such as $45^{\circ}$ and $90^{\circ}$ ). It is clearly seen that the orientation of the cylinder affect the total drag coefficient. These results are qualitatively consistent with the results for circular, elliptic and square cylinders [6-8]. The numerical value of $C_{D}$ for $n=0.3$ and $n=1.8$ for all orientation angle at low Reynolds number $R e=0.01$ are given in the Table 2 .

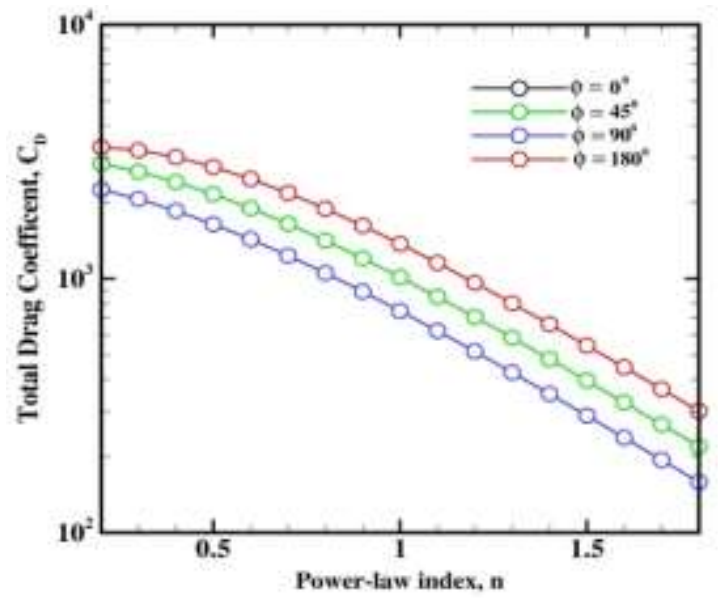

Fig. 4: Effect of orientation and power-law index on Drag coefficient. 
Table 2: Effect of orientation and power-law index on Drag coefficient.

\begin{tabular}{|c|c|c|c|c|}
\hline $\mathrm{n}$ & $\emptyset=0$ & $\emptyset=45$ & $\emptyset=90$ & $\emptyset=180$ \\
\hline 0.2 & 3289.3 & 2834.8 & 2242.6 & 32 \\
\hline 8 & 300.84 & 218.1 & 158.03 & 300.83 \\
\hline
\end{tabular}

Finally, the effect of power-law index on drag coefficient in an unambiguous manner, the normalized drag coefficient (with respect to the same Reynolds number for Newtonian fluids, $\mathrm{n}=1, C_{D} / C_{D, n=1}$ ) is shown in Fig 5. For scores of values of the power-law index. From the figure, it is confirm that for highly non-linear power-law fluids, such as shear-thinning fluids, the drag coefficient depends strongly on the shape of the body. In contrast to shear-thickening fluids with a higher value of power-law index, tend to become linear with very small viscosity[9]. It is expect that at in the shear-thickening fluids, the drag coefficient will not be sensitive to the geometrical shape of the body and this argument is supported by the present results. The argument is also consistence with argument of Whitney and Rodin [10].

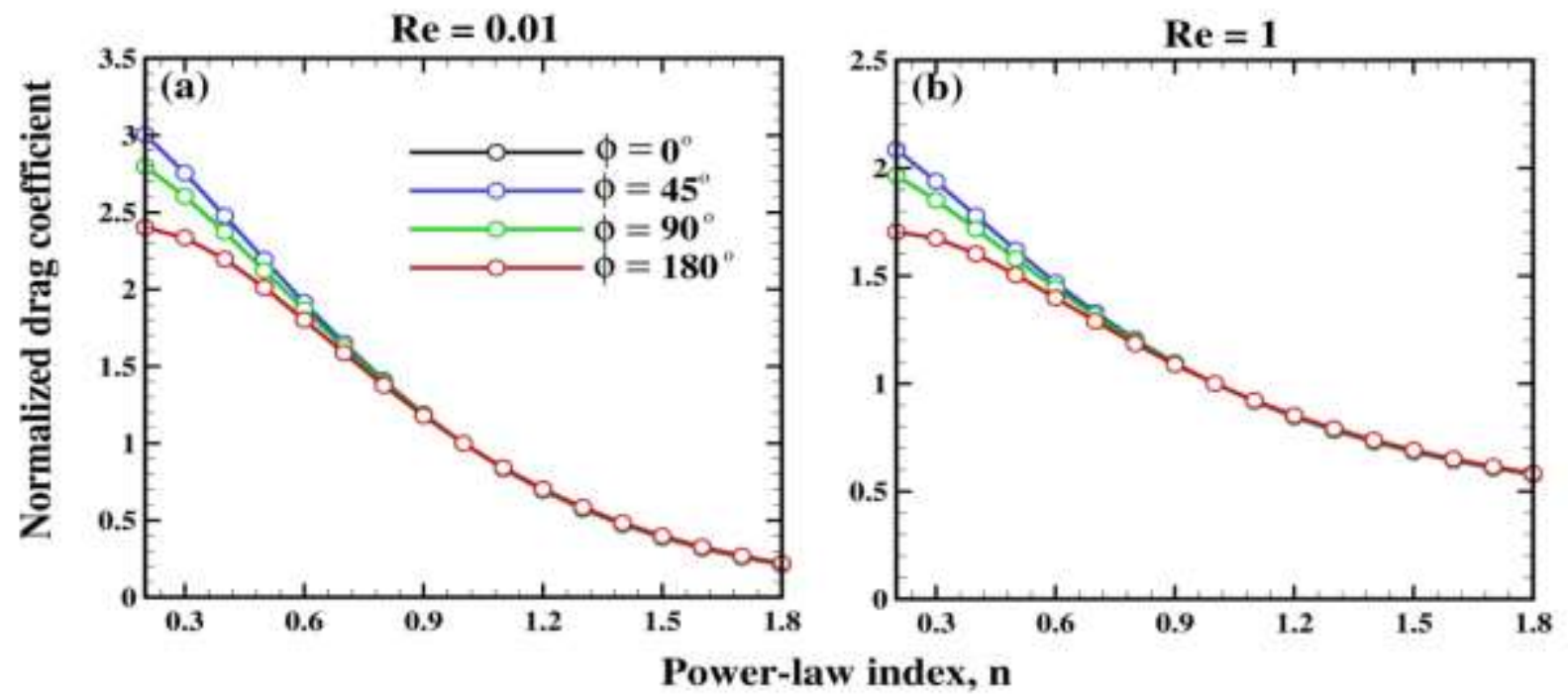

Fig. 5: Effect of orientation and power-law index on Normalized drag coefficient.

Thus, the drag coefficient is a function of Reynolds number, orientation angle with respect to incoming flow and powerlaw index. To correlate drag coefficient with Reynolds number and power-law index at different orientation angle, the data obtained is modelled to an equation of the following form

$$
C_{D}=\frac{a}{\operatorname{Re}}\left(\frac{3 n+1}{4 n}\right)^{b}
$$

Where $a$ and $b$ are the fitting parameter which can be evaluated from nonlinear regression analysis.

Table 11: Fitting parameters for correlation presented in Eq.(11).

\begin{tabular}{llllll}
\hline $\boldsymbol{\varphi}$ & $\mathbf{R}^{\mathbf{2}}$ & $\boldsymbol{a}$ & $\boldsymbol{b}$ & Max Error & Average Error \\
\hline 0 & 0.975 & 10.200 & 0.812 & 29.185 & 10.940 \\
45 & 0.979 & 10.822 & 1.004 & 30.987 & 11.319 \\
90 & 0.971 & 11.442 & 1.082 & 32.578 & 11.673 \\
180 & 0.971 & 11.426 & 1.081 & 32.669 & 11.715 \\
\hline
\end{tabular}


These equations valid at low Reynolds number, $\operatorname{Re} \leq 1$ and $0.3 \leq n \leq 1.8$ for each orientation.

\section{Conclusions}

In this present study, the flow of a non-Newtonian, power-law fluids past a semi-circular cylinder at different orientation at low Reynolds number. The streamline profile at low Reynolds number follow the shape of the body there is no flow separation observed except for $\mathrm{Re}=0.1$ for shear-thickening fluids $(\mathrm{n}>1)$. The surface shear rates is stronger in shear-thinning fluids irrespective of orientation angle. It is observed that at low Reynolds number, the variation of shear rates are much smaller in shear-thinning fluids.Finally the orientation effect is observed in shear-thinning fluids $(n<1)$ at low Reynolds number and follow the classical inverse relationship between drag coefficient and Reynolds number. On the other hand, the drag coefficient for shear-thickening fluids $(n>1)$ tend to coincide irrespective of their shape of the body.

\section{Acknowledgements}

The authors would like to Dr. C. Sasmal, Department of Chemical Engineering, Indian Institute of Technology Ropar, India for provided the software facility and guidance.

\section{References}

[1] R. P. Chhabra, J. F. Richardson, "Non-Newtonian Flow and Applied Rheology," Engineering Applications: Elsevier Science, 2011.

[2] R. B. Bird, W. E. Stewart, E. N. Lightfoot, Transport Phenomena. Wiley, 2007.

[3] COMSOL. COMSOL Multiphysics: Version 3.5a. User's guide: Comsol, 2008.

[4] A. A. Pantokratoras, "Note on the Drag Coefficient of Steady Flow of Non-Newtonian, Power-Law Fluids across Unbounded Two-Dimensional Bodies at Low Reynolds Numbers," Fluids, vol. 2, 2017.

[5] Oseen CW. Hydrodynamik: Akad. Verl.-Ges., 1927.

[6] R. P. Bharti, R. P. Chhabra, V. Eswaran, "Steady forced convection heat transfer from a heated circular cylinder to power-law fluids," International Journal of Heat and Mass Transfer, vol. 50, pp. 977-90, 2007.

[7] P. K. Rao, A. K. Sahu, R. P. Chhabra, "Flow of Newtonian and Power-Law Fluids Past an Elliptical Cylinder: A Numerical Study," Industrial \& Engineering Chemistry Research, vol. 49, pp. 6649-61, 2010.

[8] R. P. Koteswara, A. K. Sahu, R. P. Chhabra, "Momentum and heat transfer from a square cylinder in power-law fluids," International Journal of Heat and Mass Transfer, vol. 54, pp. 390-403, 2011.

[9] A. Chandra, R. P. Chhabra, "Influence of power-law index on transitional Reynolds numbers for flow over a semicircular cylinder," Applied Mathematical Modelling, vol. 35, pp. 5766-85, 2011.

[10] M. J. Whitney, G. J. Rodin, "Force-velocity relationships for rigid bodies translating through unbounded shearthinning power-law fluids," International Journal of Non-Linear Mechanics, vol. 36, pp. 947-53, 2001. 